\title{
RAÍZES SÓCIO-FILOSÓFICAS E EVOLUÇÃO DA GROUNDED THEORY
}

\author{
SOCIO-PHILOSOPHICAL ROOTS AND EVOLUTION OF GROUNDED \\ THEORY
}

\author{
Ângela Simões ${ }^{1}$ \\ Paula Sapeta ${ }^{2}$
}

\begin{abstract}
Resumo: A Grounded Theory (GT) é uma metodologia que, a partir das experiências vivenciadas por atores sociais, e através de processos de codificação e comparação constante, permite o desenvolvimento de teoria. Este ensaio teórico, de natureza descritivo-reflexiva, elaborada durante a investigação acerca da Promoção e Preservação da Dignidade no Contexto de Cuidados em Lares de Idosos, realizada no contexto do Doutoramento em Enfermagem da Universidade de Lisboa, tem o objetivo de apresentar a revisão narrativa e metodológica sobre as origens e evolução da $G T$, desde a influência fundamental do interacionismo simbólico, do pragmatismo e da Escola de Chicago salientando o momento diacritico na trajetória dos seus autores originais, Barney Glaser e Anselm Strauss, pautado por ideias distintas sobre o processo de recolha e análise de dados e sobre a postura do pesquisador perante o contexto e a própria investigação, que marcará a evolução da metodologia até à atualidade.
\end{abstract}

Palavras-chave: Investigação qualitativa; Grounded Theory; Interacionismo Simbólico.

Abstract: The Grounded Theory (GT) is a methodology that, from the experiences lived by social actors, and through processes of codification and constant comparison, allows the development of theory. This theoretical essay, of a descriptive-reflexive nature, elaborated during the investigation about the Promotion and Preservation of Dignity in the context of Care in Nursing Homes, carried out in the context of the $\mathrm{PhD}$ in Nursing of the University of Lisbon, has the purpose of presenting the narrative and methodological revision on the origins and evolution of the GT, from the fundamental influence of symbolic interactionism, pragmatism and the Chicago School emphasizing the diacritical moment in the trajectory of its original authors, Barney Glaser and Anselm Strauss, based on distinct ideas about the process of data collection and analysis and on the researcher's posture before the context and the research, that will mark the evolution of the methodology to date.

Keywords: Qualitative research; Grounded Theory; Symbolic Interactionism.

\section{Introdução}

Reconhecidamente, a tradição científica possui um histórico quantitativo, no qual a matemática foi durante muito tempo considerada a "rainha das ciências" e os estudos

\footnotetext{
${ }^{1}$ Doutor em Enfermagem pela Universidade de Lisboa (UL). Escola Superior de Saude Dr. Lopes Dias (ESALD), Instituto Politécnico de Castelo Branoc (IPCB). Investigador integrado Age.Comm - Unidade de Investigação Interdisciplinar - Comunidades Envelhecidas Funcionais, Castelo Branco, Portugal. Email: asimoes@ipcb.com

${ }^{2}$ Doutor em Enfermagem pela Universidade de Lisboa (UL). Escola Superior de Saude Dr. Lopes Dias (ESALD), Instituto Politécnico de Castelo Branoc (IPCB). Investigador integrado Age.Comm - Unidade de Investigação Interdisciplinar - Comunidades Envelhecidas Funcionais, Castelo Branco, Portugal. Email: paulasapeta@ipcb.pt
} 
de natureza menos quantificável referidos sob o rótulo pejorativo de soft science (GUBA; LINCOLN, 1994).

Quando o conhecimento sobre determinado fenómeno é escasso e não existe teorização acerca do mesmo ou pretende-se descrevê-lo de acordo com o ponto de vista do sujeito, Bortoff e Morse (1994) recomenda o uso de métodos qualitativos. O interesse e forte desenvolvimento dos estudos qualitativos pode ser atribuído ao que Flick (2004, p. 17) denomina como "pluralização das esferas da vida". De fato, o contexto de pósmodernidade tem instigado investigações voltadas para o entendimento da complexidade dos processos sociais e culturais. A velocidade das mudanças, o carácter fragmentado e multifacetado das relações têm gerado maior demanda por significados, dimensão que está além do alcance dos números.

A investigação qualitativa é a tipologia convencional, porém não exclusiva, dos estudos interpretativos e construtivistas estabelecendo-se como a forma mais adequada de promover o acesso ao campo dos significados. Ressurgiu com maior intensidade a partir do movimento reformista de cunho intelectual e crítico na academia, na década de 1970, dentro das ciências sociais, em resposta à insatisfação com os métodos experimentais e estatísticos (SCHWANDT, 2004). A preocupação da comunidade científica era desenvolver estratégias de investigação cujas orientações não visassem a explicação e/ou a generalização, privilegiando a descrição e o entendimento dos mesmos. As abordagens qualitativas podem ser sintetizadas em três posturas referentes à forma de compreensão do objeto de estudo e foco metodológico: a tradição do interacionismo simbólico, que estuda o significado atribuído às atividades e ambiente pelos indivíduos; a etnometodologia que trata da elaboração das realidades sociais e as posturas estruturalistas ou psicanalistas que partem de processos do inconsciente psicológico ou social (FLICK, 2004).

Carter e Little (2007) indicam que a investigação qualitativa tem como objetivo compreender o significado da ação humana e fazer perguntas sobre os acontecimentos no contexto em vez de provar hipóteses predeterminadas. Denzin e Lincoln (2000) destacam que a investigação qualitativa é multimetódica e implica uma abordagem interpretativa naturalista em relação ao objeto de estudo, o que significa que os investigadores estudam a realidade no seu contexto natural e tentam dar sentido, ou interpretar os fenómenos, de acordo com os significados das pessoas envolvidos. Para Taylor e Bogdan (1994) a investigação qualitativa é indutiva, inclui uma perspetiva holística do contexto, é sensível e tenta entender as pessoas. 
Miles e Huberman (1994) indicaram que a) a investigação qualitativa é realizada através do contato e permanência prolongada em campo, b) o papel do investigador é conseguir uma visão holística do contexto em estudo: a sua lógica, as suas disposições e as suas regras explícitas e implícitas; c) o investigador tenta capturar dados sobre as perceções dos atores, a partir de dentro, com atenção, compreensão empática e suspensão das perceções pessoais de objetos de discussão; d) lendo através dos materiais, o investigador pode isolar determinados temas e expressões que podem ser verificados com os participantes; e) são possíveis muitas interpretações; f) o investigador é o instrumento principal da investigação.

Neste estudo em particular, eram exigidos métodos e meios para explorar um fenómeno que pode ser obscurecido por suposições implícitas e ofuscada pela compreensão atual dos conceitos ancora. Um fenómeno complexo, multifactorial e multidimensional assente na interação social e interdisciplinaridade.

A Grounded Theory (GT) ou Teoria Fundamentada desenvolvida por Glaser e Strauss respondeu a todas as necessidades, oferece um conjunto de procedimentos estruturados, com objetivo de gerar teoria, focado na variação, elaborada complexidade e significado de processos sociais e consegue demonstrar como as condições contextuais estruturam o processo social. Permite estudar fenómenos complexos (CHARMAZ, 2009), em que se abordam questões sociais (GLASER; STRAUSS, 1967) e experiências socialmente construídas (GOULDING, 1999; CHARMAZ, 2009).

Com o objectivo conhecer as raízes sócio-filosóficas e evolução da GT foi desenvolvida uma revisão narrativa, aqui apresentada de forma simples e didática, salientado os aspetos âncora da sua história, que a justificam. Através da pesquisa bibilográfica em bases de dados, mas sobretudo, em documentos pessoais, como entrevistas e editoriais dos autores fundadores da metodologia e as suas obras mais representativas foi desenhado o percurso que se apresenta desde a a Escola de Chicago de Sociologia, da Escola de Columbia até aos estudos em conjunto de Glaser e Strauss na Universidade da Califórnia, qual lancariam as bases para a construção da GT. Finalizamos este artigo com a apresentação dos desdobramentos da várias gerações de GT, fomentados em grandes parte, pelas divergências epistemológicas dos autores originais e pelos contributos dos vários autores (seus seguidores) que marcam a diversidade e a riqueza da GT na investigação contemporânea. 


\section{Grounded Theory}

A $G T$ é uma abordagem qualitativa sistemática que enfatiza a geração de teorias de médio alcance a partir de dados a um nível formal ou substantivo (GLASER, 1978) desenvolvida por dois sociólogos, BarneyGlaser e Anselm Strauss. No âmbito do seu estudo sobre a morte de doentes terminais em contexto hospitalar, sentiram a necessidade de uma formalização metodológica não especulativa, cuidadosamente legitimada que permitisse a geração de teoria, de modo a combater o estatuto desvalorizado que as metodologias qualitativas tinham na época e reagir contra a produção de conhecimento que não é fundamentado nas necessidades ou experiências das pessoas. Propuseram por isso um modelo de investigação grounded $^{2}$ expresso na obra TheDiscoveryofGroundedTheory (1967).

[...]uma perspetiva de investigação qualitativa cujas técnicas e processos sistemáticos de análise permitem ao investigador desenvolver uma teoria substantiva que respeita os critérios para fazer "boa" ciência: significado, compatibilidade entre observação e teoria, generalização, precisão, rigor e verificação. Os procedimentos são concebidos para dar precisão e rigor ao processo de análise, sem esquecer a importância da criatividade. É a criatividade que permite ao investigador colocar aos dados questões pertinentes e estabelecer comparações que tornem visíveis novas abordagens relativamente ao fenómeno e novas formulações teóricas (STRAUSS; CORBIN, 2008, p. 31).

A $G T$, enquanto metodologia qualitativa, partilha o princípio ontológico e epistemológico que foram referidos anteriormente. Assim, apresenta algumas similaridades com outras metodologias qualitativas, nomeadamente em relação ao materiais analisados (entrevistas, observações de campo, entre outros) e o facto do groundedtheorist assumir a responsabilidade do seu papel interpretativo e da inclusão das perspetivas das vozes estudadas.

No entanto, existem também, algumas diferenças em relação a outras metodologias qualitativas. Características próprias que permitem que mantenha a sua identidade única. O desenvolvimento de teoria é uma característica particularmente importante (GLASER; STRAUSS, 1967; STRAUSS, 2003; STRAUSS; CORBIN, 2008). De acordo com Strauss e Corbin (2008, p. 13), a teoria substantiva "é uma teoria derivada dos dados recolhidos", destacando que a GT considera a estreita relação entre a recolha de dados, análise e posterior elaboração de uma teoria baseada nos dados obtidos como uma das suas características fundamentais:

\footnotetext{
2“Grounded" remete para a ideia de estudo enraizado na especificidade da realidade a investigar.
} 
[...] é descoberta, desenvolvida e provisoriamente verificada através da recolha sistemática de dados relativos ao fenómeno em estudo. Recolha de dados, análise e teoria estão reciprocamente relacionados entre si. Não se começa por uma teoria que depois se verifica. Começa-se com uma área de estudo, [área substantiva] o que for relevante para essa área irá emergir (STRAUSS; CORBIN, 2008, p. 23).

Assim, a GT permite gerar uma teoria com base nas informações fornecidas pelos atores sociais, que vivem ou estão intimamente relacionados com o problema estudado, através do qual se tenta explicar os fenómenos ou processos sociais analisadas na investigação (STRAUSS; CORBIN, 2008; TRINDAD; CARRERO; SORIANO, 2006; ANDREU; GARCÍA-NIETO; PÉREZ CORBACHO, 2007; CHARMAZ, 2009). As técnicas e os processos de análise propostos permitem ao investigador "formular interpretações teóricas dos dados fundamentadas na realidade [o que] é um poderoso meio para compreender o mundo exterior" (STRAUSS; CORBIN, 1990, p.9) em que "as questões [...] tendem a estar orientadas para a ação e processo" (STRAUSS; CORBIN, p. $38)$.

Este estudo sobre a promoção e preservação da dignidade é orientado para a ação porque procura explorar entendimentos subjetivos, tendo em conta o papel que a interação com os outros desempenha no desenvolvimento de significado (BLUMER, 1998, SCHWANDT, 2004).

\subsection{Raízes Sócio-Filosóficas da Grounded Theory}

Os problemas sociais decorrentes da industrialização e urbanização do início do século XX constituíram o estímulo para os investigadores da época desenvolverem uma perspetiva teórica distinta, voltada para o estudo sistemático do comportamento social humano. As contribuições teóricas desta perspetiva, conhecida como interacionismo simbólico, são advindas das obras de clássicos da Escola de Sociologia de Chicago como Charles Cooley, John Dewey, William Thomas e, sobretudo, George Mead, considerado como o principal precursor e inspirador do movimento interacionista (JOAS, 1999; ÁLVARO et al., 2007). Destaca-se também a influência filosófica do pragmatismo ${ }^{3}$ sobre os estudiosos da Escola de Chicago. Podemos afirmar que o pragmatismo de James e

\footnotetext{
${ }^{3} \mathrm{O}$ pragmatismo constitui uma escola de filosofia estabelecida no final do século XIX associada a Peirce, James, Dewey e Mead, que vai contra as reflexões filosóficas abstratas e insuficientes, princípios fixos e absolutos, sistemas fechados e grandes teorias. A investigação que defendem, deve procurar a verdade e servir apenas o que realmente faz diferença na vida prática. Defendem que as ideias verdadeiras são as que podemos assimilar, validar, corroborar e verificar (JAMES, 1907). Para os pragmatistas, o conhecimento existe na forma de afirmações ou teorias as quais são vistas como instrumentos ou ferramentas (BRYANT, 2009), por isso, é também comum o uso da expressão instrumentalismo.
} 
Dewey foram a corrente filosófica que inspirou e orientou as escolhas políticas, teóricas e metodológicas dos autores da Escola de Chicago, e o interacionismo simbólico de Mead a sua principal e mais duradoura produção teórica.

A relação entre a $G T$ clássica (GLASER; STRAUSS, 1967) e a tradição interpretativa do interacionismo simbólico é forte e histórica. Por isso é correto afirmar que as raízes sócio filosóficas da $G T$ estendem-se pelo pragmatismo, Escola de Chicago e Interacionismo simbólico. A influência dessas correntes na proposição metodológica da $G T$ aparece de duas formas. Primeiro, pela rejeição da ideia de que a verdade científica reflete uma realidade externa e independente, ao afirmar que esta resulta tanto do ato de observação como do consenso emergente dentro de uma comunidade de observadores. Em segundo lugar, pela ênfase no simbólico e no caráter social do pensamento e do comportamento humano, contrária às posições mais objetivistas. A proposta fundamental da $G T$ é colocar o processo interpretativo através do qual os indivíduos apreendem uma realidade subjetiva no centro da investigação científica social.

Segundo Joas (1999) Cooley foi o primeiro a proclamar a necessidade de um pragmatismo "social" ou "sociológico" e o primeiro a desenvolver uma teoria do Eu. Entretanto, a análise pragmática de situações de interação social e autorreflexão individual foi creditada a Mead. As conceções apresentadas por Mead são consideradas a fonte principal da qual derivaram as interpretações que deram origem ao interacionismo simbólico que começou a delinear-se entre os anos de 1930 e 1940.

Mead estudou a ação interpessoal, indicando que o modo de ação de um indivíduo suscita reações do seu parceiro, tornando-se condição para a continuidade das suas próprias ações. A ação de cada um só adquire sentido através da ação do outro e durante o processo de qualquer ato social, os objetos do ambiente percebido definem-se redefinem-se. De tal dinamismo resulta a interação simbólica, a qual não se dá por reação direta às ações e gestos do outro, mas, mediante uma interpretação destas ações ou gestos com base no significado que lhes é atribuídopresente na conduta social, em que emergem os símbolos significantes. Só quando o indivíduo se identifica com tais símbolos, se torna consciente a significação (MEAD, 1967).

Afirma que a mente é uma relação do organismo com a situação, que se realiza por meio de uma série de símbolos. Quando um determinado gesto representa a ideia que há por trás de si e provoca essa ideia no outro indivíduo, obtém-se um símbolo significante. No momento em que tal gesto promove uma reação adequada do outro indivíduo, tem-se um símbolo que responde a um significado na experiência do primeiro 
indivíduo e que também evoca esse significado no segundo indivíduo. Assim, a base da significação está presente na conduta social, em que emergem os símbolos significantes. Só quando o indivíduo se identifica com tais símbolos, se torna consciente a significação (MEAD, 1967).

Para explicar este processo de interação que envolve definição e redefinição de objetos do ambiente percebido, Mead introduziu as noções de Self, Eu e Mim. A característica da pessoa como objeto para si está representada pelo termo Self (si mesmo), e este Self permite que o indivíduo interaja socialmente consigo mesmo, da mesma forma que ele interage socialmente em relação a outras pessoas. "Quando não só se escuta a si, mas também se responde [...] tão realmente como se responde a outra pessoa, então temos uma conduta em que os indivíduos se convertem em objetos para si mesmos" (MEAD, 1967, p. 171).

A sociedade representa o contexto dentro do qual o Self surge e se desenvolve. Mead esclarece que o Self representa um processo social no interior do indivíduo, que envolve duas fases analíticas distintas: o "Eu", que é a tendência impulsiva do indivíduo e o "Mim", que representa o outro generalizado. É na forma do outro generalizado que os processos sociais influenciam a conduta dos indivíduos envolvidos e a comunidade exerce controlo sobre o comportamento dos seus membros individuais (MEAD, 1967).

Acrescenta, ainda, que o progresso social humano implica o emprego, por parte dos indivíduos, do seu mecanismo de consciência de si, socialmente surgido. Este pode ser usado tanto para produzir as transformações sociais progressivas, como para o desenvolvimento de si mesmos ou de suas personalidades individuais, como forma de se manter adaptativo a esse ritmo de reconstrução social (MEAD, 1967).

O movimento do interacionismo simbólico teve o seu nome cunhado em 1937 por Herbert Blumer, que estabeleceu os pressupostos da abordagem interacionista.Sustentando-se nos preceitos teóricos de Mead, Blumer (1998) reafirma a noção de que o significado é um produto social, uma criação que emana das atividades dos indivíduos à medida que estes interagem. Esclarece que a natureza do interacionismo simbólico tem como base a análise de três premissas. O ser humano orienta os seus atos em direção às coisas em função do que estas significam para ele; o significado das coisas surge como consequência da interação social que cada qual mantém com o seu próximo; os significados manipulam-se e modificam-se mediante um processo interpretativo desenvolvido pelo individuo ao defrontar-se com as coisas que vai encontrando no seu caminho. 
O tema central que aborda são os processos de interação social, ação social caracterizada por uma orientação imediatamente recíproca. No entanto, o conceito específico de interação privilegia o caráter simbólico da ação social. Assim, de acordo com Blumer (1998), as relações sociais são vistas como algo aberto e subordinado ao reconhecimento contínuo por parte dos membros da comunidade.

Para os interacionistas simbólicos o significado é um dos mais importantes elementos na compreensão do comportamento humano, das interações e dos processos. Argumentam que, para alcançar uma compreensão plena do processo social, o investigador precisa apoderar-se dos significados que são experienciados pelos participantes num contexto particular. Blumer (1998) critica metodologias com paradigmas positivistas por utilizar meios de estabelecer a validade empírica de certos estudos que ele julga inadequados para captar o caráter específico do objeto de estudo. Propõe, então, uma posição metodológica para o interacionismo simbólico que defende a noção de que para compreender o mundo é necessário analisá-lo em termos das ações e interações dos seus participantes. Defende que o investigador deve ser capaz de interagir ativamente com as pessoas que estuda, de modo a ver o fenómeno do ponto de vista destas e no seu contexto natural. Por conseguinte, ao adotar a abordagem interacionista, o investigador precisa estar ativamente imerso no mundo em estudo e empreender uma análise das suas partes fundamentais:

\begin{abstract}
o estudo exploratório é o meio para conseguir um conhecimento extenso e profundo da esfera da vida social e desenvolver e acentuar a sua investigação. [...] Por seu caráter reflexivo, não está sujei-to a nenhum conjunto de técnicas em particular, pode recorrer à observação direta, entrevistar pessoas, obter informações sobre a vida real, utilizar cartas e diários, consultar documentos públicos e organizar discussões de grupo. A finalidade da investigação exploratória é traçar um quadro em estudo, tão completo e preciso quanto permitem as condições vigentes [...]. A inspeção consiste em examinar o elemento analítico dado, considerando-o de diferentes ângulos, estabelecendo diversas perguntas e examinando novamente à luz das mesmas; em outras palavras, um exame detido e profundo. A exploração e a inspeção representam a descrição e a análise e correspondem ao que se denomina investigação naturalista; um processo destinado a abordar o mundo empírico no seu caráter natural e contínuo, em lugar de se limitar a uma simulação do mesmo, uma abstração ou a sua substituição por uma imagem pré-estabelecida (BLUMER, 1998, p. 33).
\end{abstract}

As ideias centrais do interacionismo simbólico são de que, individualmente e coletivamente, os seres humanos são ativos, criativos e não somente reativos às forças do ambiente externo. Os ambientes nos quais eles agem e com os quais interagem são simbólicos; os símbolos são produzidos em interação e podem ser manipulados no curso da interação; o pensamento pode ser usado para antecipar a efetividade de alternativas de 
ação planeadas para resolver problemas e a escolha entre cursos de ação alternativos é uma característica da conduta social (STRYKER; VRYAN, 2006).

As duas variantes mais proeminentes do interacionismo simbólico são representadas pelas Escolas de Chicago e Iowa associadas, respetivamente, às figuras de Herbert Blumer e ManfordKuhn. Os pressupostos básicos do interacionismo simbólico, elaborados por Blumer, são conhecidos como Interacionismo Clássico ou Interacionismo da Escola de Chicago que influenciou indubitavelmente BarneyGlaser e Anselm Strauss.

O objetivo principal da GT é compatível com o objetivo global de interacionismo simbólico, ou seja, fornecer uma teoria que explora o comportamento humano, uma abordagem para estudar a conduta humana e a vida em grupo. De acordo com Glaser (1978, p. 100), o principal objetivo da GT é descobrir o processo(s) social básico, isto é,

as reflexões e resumos teóricos dos fluxos padronizados, uniformes e sistemáticos da vida social que as pessoas percorrem, e que podem ser conceptualmente "capturado" e posteriormente compreendido através da construção de teorias de processos sociais básicos.

A $G T$ e o Interacionismo Simbólico não são apenas consistentes nos seus objetivos, mas também nos seus pressupostos. Nos pressupostos ontológicos, que se referem à natureza da realidade e do que os seres humanos podem saber sobre ela (LINCOLN; GUBA, 2000), os investigadores que adotam o interacionismo simbólico e a GT consideram que os seres humanos existem num mundo de significados simbólicos compartilhados. Em relação aos pressupostos epistemológicos, que se referem à natureza da relação entre o conhecedor e o que pode ser conhecido (GUBA; LINCOLN, 1994) para o interacionismo simbólico e a $G T$, o investigador e os participantes estão ligados de forma interativa, num relacionamento mútuo no contexto natural para poder investigar o seu comportamento. Os pressupostos metodológicos, que se referem a como o investigador pode descobrir a experiência social, como é criada, e como dá sentido à vida humana (GUBA; LINCOLN, 1994) para o interacionismo simbólico e a $G T$, os seres humanos e os significados compartilhados da realidade só podem ser definidos através da interação com e entre o investigador e participantes no contexto dos fenómenos de interesse.

\subsection{Evolução da Grounded Theory}

Glaser e Strauss iniciaram a sua parceria na Universidade da Califórnia, San Francisco, onde desenvolveram investigação com doentes terminais. Como resultado dos seus estudos publicaram Time for Dying e Awareness of Dying e dessa experiência 
conjunta, construíram as bases do que descreveram como uma nova abordagem de investigação científica, a que chamaram Grounded Theory. É possível identificar três bases metodológicas fundamentais subjacentes à proposta original de Glaser e Strauss: a amostragem teórica, o método de comparação constante e a geração de teoria a partir de dados obtidos no âmbito da investigação.

Ao longo do tempo tem havido várias alterações à abordagem da GT. Isto devese em grande parte ao facto dos dois autores originais chegarem a um momento diacrítico sobre os objetivos, princípios e procedimentos associados à aplicação do método. Esta bifurcação, foi marcada em grande medida pela publicação em 1990 de Basics of qualitative research: grounded theory. Procedures and techniques de Strauss e Corbin que provocou acusações de distorção e infidelidade para com os objetivos centrais do método (GLASER, 1992).

As diferenças entre Strauss e Corbin (1990) e Glaser (1978, 1992) podem ser resumidas em três pontos. Em primeiro lugar diferenças processuais. Strauss e Corbin (1990) defendem o uso de procedimentos adicionais, tais como, "agitando a bandeira vermelha", "o flip-flop" e o uso de "matrizes" para maximizar a sensibilidade teórica e assegurar o desenvolvimento de um teoria enraizada nos dados que seja densa e precisa (STRAUSS; CORBIN, 1990, 2008). Glaser (1992) desafia estas técnicas processuais adicionais argumentando que o método de comparação constante é suficiente, em si mesmo, para desenvolver uma teoria.

Em segundo lugar a postura do investigador em relação aos dados. A abordagem de Strauss e Corbin (1990, 2008) permite a inclusão da experiencia e interpretação do investigador, uma vez que a codificação de dados é essencialmente um processo criativo e alega que a experiência e conhecimento anterior são benéficos para o desenvolvimento da teoria. Em contraste, Glaser (1992) argumenta que não se deve abordar os dados com ideias preconcebidas, e o uso de literatura deve ser adiada até que a teoria tenha sido desenvolvida.

Em terceiro lugar as diferenças filosóficas entre os autores. As técnicas processuais divergentes podem ser rastreadas pelas perspetivas filosóficas (ANNELLS, 1997a, CHARMAZ, 2009) que podem decorrer da sua formação anterior e influências intelectuais. Glaser com uma influência quantitativa e positivista e Strauss com influências qualitativas e construtivistas. A análise contemporânea é que The Discovery of Grounded Theory foi uma tentativa de sintetizar o melhor das duas tradições académicas das quais provinham, no entanto não significou uma integração entre as duas 
escolas de pensamento mas apenas uma interseção entre as suas áreas de interesse. Glaser teve uma formação em filosofia analítica e investigação quantitativa na Universidade de Colúmbia. A sua formação enfatizou a investigação empírica em conjunto com o desenvolvimento da teoria. Strauss veio da Escola de Chicago, seriamente influenciado por Herbert Blumer e Everett C. Hughes e os seus métodos pragmáticos de trabalho de campo. A abordagem de Strauss é baseada no pragmatismo americano, especialmente nas ideias de Peirce e na visão instrumentalista do processo de investigação de John Dewey (BRYANT, 2009).

Segundo Babchuk (2010) há um abismo epistemológico e metodológico entre as versões da $G T$ abraçadas por Glaser e por Strauss. Afirma que Glaser citado por Babchuk (2010, p. 2):

[...] parece ver a GT como um tipo de método mais laissez-faire, inerentemente flexível e guiada primordialmente pelos informantes e as suas realidades socialmente construídas. O mundo dos informantes deve emergir naturalmente da análise com pequeno esforço ou atenção detalhada ao processo, por parte do investigador.

Strauss mostrou-se interessado no desenvolvimento de um conjunto de métodos para a realização de investigação. Torna-se dogmático em relação às técnicas de codificação altamente complexas e sistemáticas e defendia uma perspetiva construtivista. Fez modificações significativas, por vezes, descartando "paradigmas" por inteiro a favor de procedimentos mais abertos (STRAUSS; CORBIN, 2008). Glaser discordou de muitas das metodologias específicas, que considerou "forçar" os dados em estruturas preexistentes (GLASER, 1992), com uma perda potencial da capacidade ("sensibilidade") para discernir e criar novas teorias (GLASER, 1978). Glaser salienta a natureza da interpretação contextual e emergente de desenvolvimento da teoria, numa perspetiva objetivista.

A partir desse ponto a $G T$ dividiu-se em dois campos, cada um subtilmente distinguido pelos seus próprios procedimentos ideográficos e cria-se, o que é conhecido como escolas ou correntes "Glaseriana" e "Strausseriana" (COONEY, 2010; JONES; ALONY, 2011) que enfatizam respetivamente, por um lado, o gerar teoria a partir dos dados produzidos pela própria investigação e, por outro, o foco na descrição e análise de dados utilizando novos instrumentos, tais como codificação axial ou a utilização de software informático (CLARKE, 2003; RAYMOND, 2005; VALLES, 2005; TRINIDAD; CARRERO; SORIANO, 2006; ANDREU; GARCÍA-NIETO; PÉREZ 
CORBACHO, 2007; CHARMAZ, 2009; COONEY, 2010; ESCALANTE, 2011; JONES; ALONY, 2011; HALL; GRIFFITHS; MCKENNA, 2013).

É importante notar que, ao longo dos anos, a GT na versão original tem recebido contribuições significativas de vários autores, com os alunos dos dois fundadores a desenvolveram as suas próprias práticas e as suas próprias orientações filosóficas, o que permitiu o surgimento de diferentes perspetivas e ênfase sobre a geração de teoria (GOULDING, 1999; ANDREU, GARCÍA-NIETO, PÉREZ CORBACHO, 2007; ALLEN, 2010; COONEY, 2010; JONES; ALONY, 2011). Após a descoberta inicial da GT (GLASER; STRAUSS, 1967) e das divergências entre os autores originais, duas grandes orientações surgiram (BABCHUK, 2010; KELLE, 2005), seguidas por uma "segunda geração" de groundedtheorists que criativamente estenderam e recombinaram uma ou ambas as orientações principais (MORSE et al., 2009) ou estabeleceram outras ramificações.

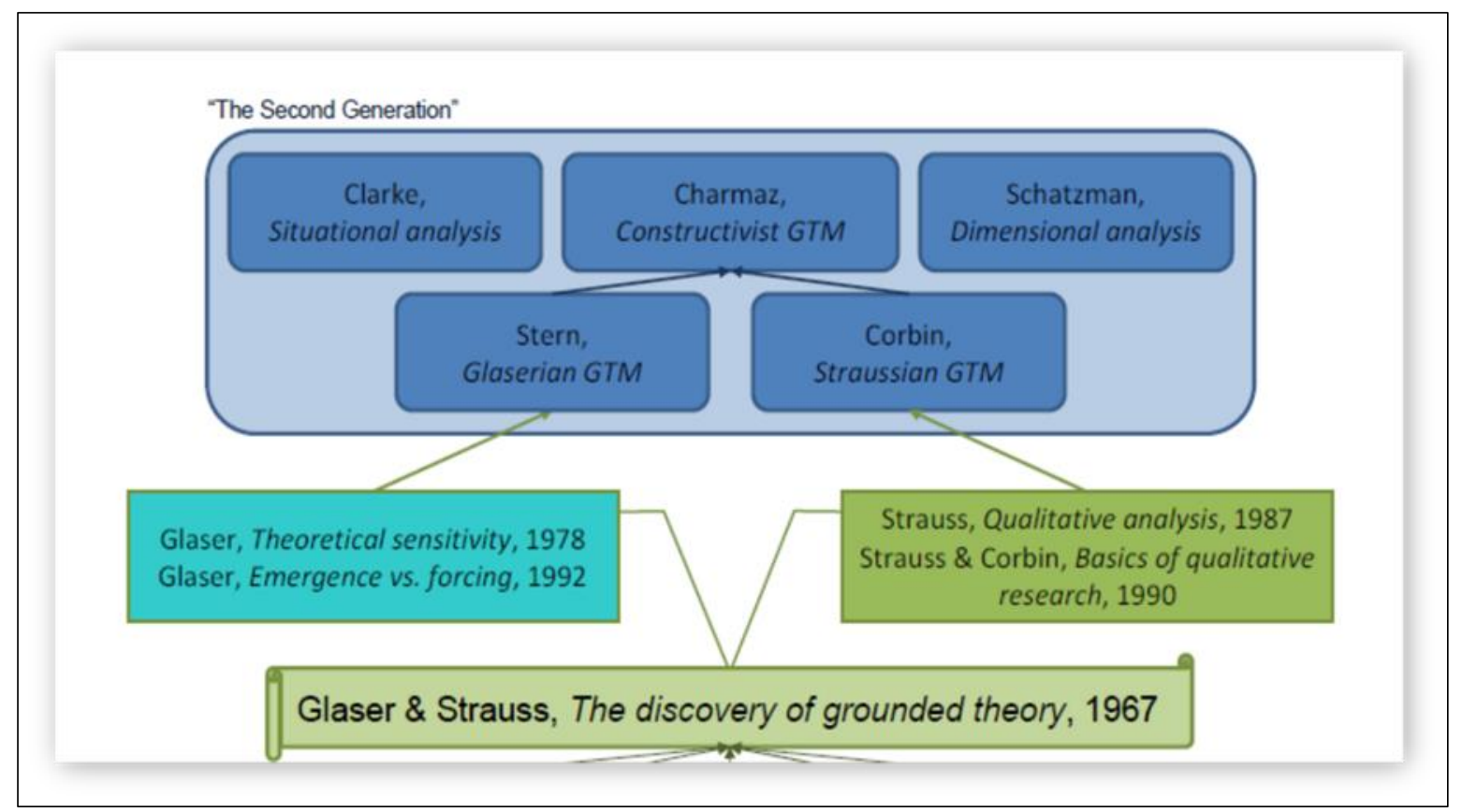

Figura 1: Gerações da Grounded Theory

Fonte: Muller (2014)

A polémica entre Glaser e Strauss fez aflorar diferentes maneiras de ver e fazer ciência social. Se as duas tradições, de Colúmbia e Chicago, se uniram na década de 60 para lançar The Discovery of Grounded Theory, as diferenças de visão do mundo acabaram por separar Glaser e Strauss. O debate entre os dois "pais" da GT não cessou e as discussões sobre a $G T$ continuam, com o método a ganhar novas leituras. Como resultado a metodologia tem contribuído para operacionalizar trabalhos tanto de cunho 
mais fenomenológico como mais positivista, existindo autores como Annells (1997b) que apontam para a possibilidade de desenvolver vertentes dialéticas para $a G T$.

O falecimento de Strauss, em 1996, fez com que o debate se tornasse menos acirrado. O seu último trabalho, organizado em parceria com Juliet Corbin foi o livro Grounded Theory in Practice, publicado em 1997. Glaser, por sua vez, através do seu instituto de investigação (The Grounded Theory Institute) continua a promover discussões em redor da metodologia.

Alguns autores da "segunda geração" apresentam estratégias e diretrizes diferentes para a recolha de dados e processo de codificação (GOULDING, 1999; CHARMAZ, 2009; PARTINGTON, 2000; PATTON 2002; STRAUSS; CORBIN, 1990, 2008). As modificações no processo e os métodos de codificação criaram um aceso debate entre os grounded theorists, que se mantem até hoje, com os defensores da "primeira geração" a alertar para o facto de que estruturas menos rígidas e sistemáticas no processo de codificação podem criar limitações na capacidade de completar a análise e que as mudanças na codificação são muito mais profundas do que apenas um processo de codificação diferente, são uma fratura dos elementos centrais da GT clássica (GLASER, 2013).

A abordagem de Charmaz (2009), uma das mais citadas na investigação contemporânea, juntamente com a de Glaser e a de Strauss e Corbin, reconhece que a realidade e os fenómenos estudados são construções coletivas, apelando fortemente à tradição interpretativa e construtivista, afastando-se claramente das bases objetivistas da abordagem clássica dos fundadores da GT, principalmente da abordagem Glaseriana, mas criticando também a referência pós-positivista de Strauss, no seu procedimento sistemático de codificação. Charmaz (2009), apesar de apoiar os princípios do Interacionismo Simbólico e do Pragmatismo, propõe a recuperação do ênfase no simbólico, no significado, na linguagem, na interpretação e na interação, vinculando a GT ao paradigma construtivista.

\section{Conclusão}

O presente ensaio teórico não teve a pretensão de apresentar exaustivamente a GT e a forma de operacionalização da metodologia contudo, mas apresentar as suas bases históricas, de forma simplificada e didática, possibilitando àqueles que pretendem utilizála, uma melhor compreensão das suas raízes, os seus fundamentos. A escolha da versão 
da GT a adoptar estará sempre relacionada com a forma de ver o mundo, própria e única, de cada investigador.

A GT é um referencial metodológico que possibilita a construção de conhecimento de realidades pouco exploradas. Permite um novo olhar sobre a realidade e, particualmente para a disciplina de Enfermagem, permite a geração de teorias a partir da prática, fortemente marcada pelas interações humanas.

Apesar da actual diversidade, na forma de abordar a metodologia, conforme a escolha da escola a seguir, os diferentes autores partilham e defendem a não existência de uma verdade absoluta sobre a realidade externa, que pode ser descoberta, descrita e medida, mas a valorização do contexto em que os fenómenos ocorrem.

\section{Referências}

ALLEN, L. A critique of four Grounded Theory texts. The Qualitative Report, Nova Southeastern University, v. 15, n. 6, p. 1606-1620, 2010.

ALVARO, J. et al. Introducción a la psicología social sociológica. 1. ed. Barcelona: Editorial UOC, 2007.

\section{ANDRÉU, J.; GARCÍA-NIETO, A.; PÉREZ CORBACHO, A. Evolución de la Teoría} Fundamentada como técnica de análisiscualitativo. 1. ed. Madrid: CIS, 2007.

ANNELLS, M. Grounded Theory method, part 1: within the five moments of Qualitative research. Nursing Inquiry, Carlton, v. 4, n. 2, p. 120-129, june. 1997a.

ANNELLS, M. Grounded Theory method part 2: options for users of the method. Nursing Inquiry, Carlton, v. 4, n. 3, p.176-180, aug. 1997b.

BABCHUK, W. Grounded theory as a "family of methods": A genealogical analysis to guide research. US-China Education Review, Valley Cottage, v. 8, n. 9, p.10-16. 2010.

BLUMER, H. Symbolic Interactionism. Perspective and Method. 1. ed. Berkeley: University of California Press, 1998.

BORTOFF, J.; MORSE, J. Identify types of attending: patterns of Nursing Work. The Journal of Nursing Scholarship, Medford, v. 26, p. 53-60. 1994.

BRYANT, A. Grounded Theory and Pragmatism: The Curious Case of Anselm Strauss. Qualitative Social Research, Berlim, v. 10, n. 3, p. 1-31, sept. 2009. Disponível em: http://www.qualitative-research.net/index.php/fqs/article/view/1358/2850. Acesso em: 10 out. 2013.

CARTER, S.; LITTLE, M. Justifying Knowledge, justifying method, taking action: epistemologies, methodologies, and methods of qualitative research. Qualitative Health Research, Thousand Oaks, v. 10, n. 17, p.1316-1328, sept. 2007.

CHARMAZ, K. A Construção da Teoria Fundamentada. 3. ed. Porto Alegre: Artmed, 2009. 
CLARKE, A. Seeing the person behind the patient: enhancing the care of older people using a biographical approach. Journal of Clinical Nursing, Oxford; Boston, v. 12, n. 5, p. 697-706, sept. 2003.

COONEY, A. Rigor and Grounded Theory. Nurse Researcher, Harrow, Middlesex, v. 14, n. 4, p. $17-22,2010$.

DENZIN, N.; LINCOLN, Y. (Eds.) Handbook of Qualitative Research. 2. ed. California: SAGE Publications, 2000.

ESCALANTE, E. Revisitando la crítica a la teoría fundamentada (GroundedTheory). Poliantea, Institución Universitaria Politécnico Grancolombiano, v. 7, n.12, p. 59-77, mar. 2011.

FLICK, U. Introducción a la InvestigaciónCualitativa. 1. ed. Madrid: Ediciones Morata S. L., 2004.

GLASER, B. Theoretical sensitivity: advances in the methodology of grounded theory. 1. ed. Mill Valley, CA: Sociology Press, 1978.

GLASER, B. Basics of grounded theory analysis. 1. ed. Mill Valley, CA: Sociology Press, 1992.

GLASER, B. Introduction: Free Style Memoing. The Grounded Theory Review, Charleston, v. 12, n. 2, s.p., dec. 2013. Disponível em:

http://groundedtheoryreview.com/2013/12/22/introduction-free-style-memoing/. Acesso em: 23 out. 2014.

GLASER, B.; STRAUSS, A. The Discovery of Grounded Theory: Strategies for Qualitative Research. 1. ed. Chicago: Aldine, 1967.

GOULDING, C. Grounded Theory: some reflections on paradigm, procedures and Misconceptions. Working Paper Series. United Kingdom: University of Wolverhampton, 1999.

GUBA, E.; LINCOLN, Y. Competing paradigms in qualitative research. In: DENZIN, N.; LINCOLN, Y. (Eds.). Handbook of qualitative research. 1. ed. Thousand Oaks, CA: Sage, 1994. p. 105-117.

HALL, H.; GRIFFITHS, D.; MCKENNA, L. From Darwin to constructivism: the evolution of grounded theory. Nurse Researcher, Harrow, Middlesex, v. 20, n. 3, p.17-21, jan. 2013.

JAMES, W. Pragmatism. 1. ed. New York: Vintage Books, 1907.

JOAS, H. Interacionismo simbólico. In: GUIDDENS, A.; TURNER, J. Teoria social hoje. 1. ed. São Paulo: Unesp, 1999. p.127-174.

JONES, M.; ALONY, I. Guiding the use of Grounded Theory in Doctoral studies - an example from the Australian film industry. International Journal of Doctoral Studies, University of Wollongong, v. 6, p. 95-114. 2011. Disponível em:

http://ro.uow.edu.au/cgi/viewcontent.cgi?article=1842\&context=commpapers. Acesso em: 23 nov. 2015.

KELLE, U. "Emergence" vs. "Forcing" of Empirical Data? A crucial problem of "Grounded Theory" reconsidered. Forum: Qualitative Social Research, Berlim, v. 6, n. 2, p. 1-22, may. 2005. 
LINCOLN, Y.; GUBA, E. Paradigmatic controversies, contradictions, and emerging confluences. In: DENZIN, N.; LINCOLN, Y. (Eds.). The handbook of qualitative research. 2. ed. London: Sage, 2000. p. 163-188.

MEAD, G. Mind. Self and Society. 1. ed. Chicago: The University of Chicago Press, 1967.

MILES, M.; HUBERMAN, M. Qualitative date analysis: an expanded source book. 2. ed. London: Thousand Oaks SAGE, 1994.

MORSE, J. et al. Developing grounded theory: The second generation. 1. ed. Walnut Creek, CA: Left Coast Press, 2009.

MULLER, M. Curiosity, Creativity, and Surprise as Analytic Tools: Grounded Theory Method. In: OLSON, J.; KELLOGG, W. (Eds.). Ways of Knowing. 1. ed. New York: HCI, Springer Science \& Business Media, 2014. p. 25-48.

PARTINGTON, D. Building Grounded Theories of Management in action. British Journal of Management, Londres, v.11, n. 2, p. 91-102, june. 2000.

PATTON, M. Qualitative evaluation and research methods. 3. ed. Newbury Park, CA: Sage. 2002.

RAYMOND, É. La TeorizaciónAnclada (GroundedTheory) como Método de Investigación en Ciencias Sociales: en la encrucijadade dos paradigmas. Cinta de Moebio, Santiago, Chile, v. 23, p. $217-227,2005$. Disponível em:

<http://www.facso.uchile.cl/publicaciones/moebio/23/raymond.htm>. Acesso em: 23, nov. 2015.

SCHWANDT, T. Three Epistemological Stances for Qualitative Inquiry. In: DENZIN, N.; LINCOLN, Y. (Eds.). Handbook of Qualitative Research. 2. ed. Thousand Oaks: SAGE Publications, 2004. p. 189-213.

STRAUSS, A. Qualitative analysis for social scientists.1.ed. San Francisco: Cambridge University Press, 2003.

STRAUSS, A.; CORBIN, J. Basics of qualitative research: grounded theory. Procedures and techniques. 1. ed. London: Sage Publications, 1990.

STRAUSS, A.; CORBIN, J. Basics of Qualitative Research. Techniques and Procedures for Developing Grounded Theory. 3. ed. London: Sage Publications, 2008.

STRYKER, S.; VRYAN, K. The symbolic interactionist frame. In: DELAMATER, J. (Org.). Handbook of social psychology. 1. ed. New York: Springer, 2006. p. 3-28.

TAYLOR, S.; BOGDAN, R. Introducción a los métodos cualitativos de investigación. La búsqueda de significados. 2. ed. Buenos Aires: Ediciones PAIDOS, 1994.

TRINIDAD, A.; CARRERO, V.; SORIANO, R. Teoría fundamentada GroundedTheory. La construcción de la Teoría a través de la análisis interpretacional. 1.ed. Madrid: CIS, 2006.

VALLES, M. Metodología y tecnologíacualitativas: actualización de un debate, desde la mirada más atenta en la obra de Barney G. Glaser. EMPIRIA Revista de Metodología de Ciencias Sociales, Madrid, v. 9, p.145-168, ene./jun. 2005.

Recebido em: 06 de outubro de 2017.

Aceito em: 11 de dezembro de 2018. 